NISTIR 6578

\title{
Internet Commerce for Manufacturing Data Staging
}

\section{J. G. Nell \\ C. H. Parks}

U. S. DEPARTMENT OF COMMERCE Technology Administration

Manufacturing Engineering Systems Integration Division

National Institute of Standards and Technology

Gaithersburg, MD 20899

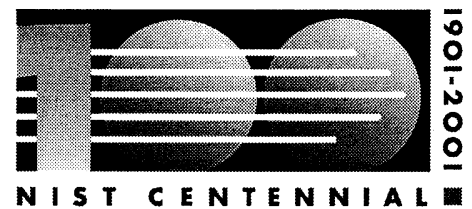

\section{NIST}

National Institute of Standards and Technology

Technology Administration

U.S. Department of Commerce 
NISTIR 6578

\section{Internet Commerce for Manufacturing Data Staging}

\section{J. G. Nell C. H. Parks}

U. S. DEPARTMENT OF COMMERCE Technology Administration

Manufacturing Engineering Systems Integration Division

National Institute of Standards and Technology

Gaithersburg, MD 20899

November 8, 2000

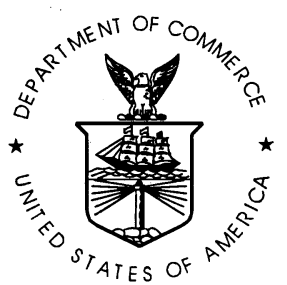

U.S. DEPARTMENT OF COMMERCE Norman Y. Mineta, Secretary

TECHNOLOGY ADMINISTRATION Dr. Cheryl L. Shavers, Under Secretary of Commerce for Technology

NATIONAL INSTITUTE OF STANDARDS AND TECHNOLOGY

Raymond G. Kammer, Director 


\section{Internet Commerce for Manufacturing Data Staging}

J. G. Nell

+1301975 5748, nell@nist.gov

Building 220/Room A131

Curtis Parks

+1 3019753517 , cparks@nist.gov

Building 225/Room A55

National Institute of Standards and Technology

Gaithersburg MD, USA, 20899

\section{Foreword}

Goods and services traded over the Internet will reach a total value of $\$ 327$ billion by 2002 , according to a recent report from Forrester Research in Cambridge, Mass. Today, most of this electronic commerce (e-commerce) on the Internet is from business-to-consumer (B2C) retail sales rather than from business-to-business (B2B) transactions among business-trading partners. In the future, however, $\mathrm{B} 2 \mathrm{~B}$ is expected to become the major form of e-commerce on the Web through the formation of virtual-trading-partner enterprises because business efficiency will be improved. This goal can only be realized if information can be exchanged and shared among business enterprises and trading partners to enable business transactions to occur reliably and safely. In particular, the manufacturing industry requires a more coordinated exchange of both business and product information across an extensive supply chain of trading partners that span a product-ordering-, manufacturing-, and distribution-life cycle measured in months, weeks, and, sometimes, days.

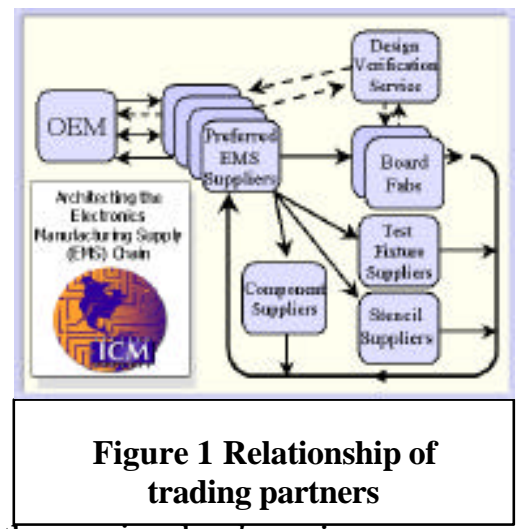

This B2B, e-commerce scenario is quite different and more demanding than a simple shopping-cart scenario found in $\mathrm{B} 2 \mathrm{C}$ retail sales. It involves a highly dynamic and variable set of business processes, data exchanges, and sequence of operations, among trading partners that require industry to use agreed-upon interfaces, protocols, and standards that can enable trading partners to exchange information across enterprises. This, in turn, requires each of the enterprises to understand its business processes and data flows, and to represent these in an electronic form to which other enterprises can receive, understand, and respond.

This paper describes work done by the National Institute of Standards and Technology (NIST) under the Internet Commerce for Manufacturing (ICM) project. A task of this project is to model selected business processes and data flows within and across trading partners involved in the manufacturing of electronic-printed-circuit boards and assemblies (See Figure 1). Appendix A of this paper contains background information about the ICM project. Figure 2 depicts the plan for deriving the models described in this paper. 
The ICM work toward developing message and data transfer in a printed-circuit-assembly-supply chain requires accurate knowledge of the process events, when they occur, and the timing of messages about those events between enterprises. We analyzed the process events to uncover the data and messages flows among the processes. The process events build upon and specialize the information in the ICM Activity Model [5] to form the Process-Flow Model. The Process-Flow Model can be linked back to the elements of the ICM Activity Model.

Process-flow models are a common element in many of the projects in both the Manufacturing Engineering Laboratory, and the Electronic Information Technologies Group of the Electronics and Electrical Engineering Laboratory. To create the Process-Flow Model we have used common software-flow-charting techniques that are easily interpreted and edited during the evolution of the ICM test bed.

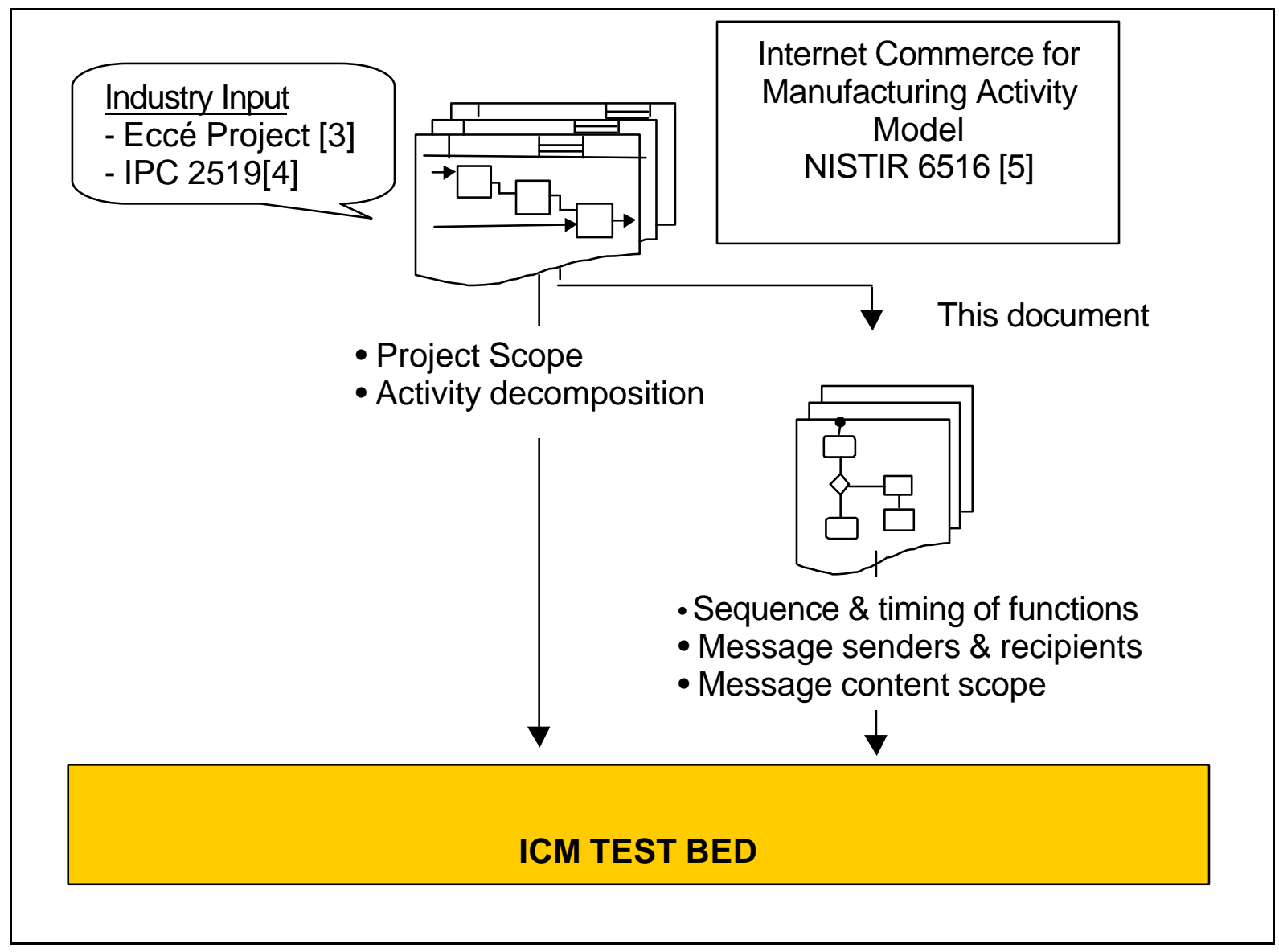

Figure 2 ICM Model Heritage

\section{Executive Summary}

This paper presents and describes a proposed process-flow-reference model for the Internet Commerce for Manufacturing (ICM) project, part of the NIST National Advanced Manufacturing Test Bed program. 
The portion of the printed-circuit-assembly-supply chain from the time when the design is complete until receiving the completed assembly comprises mostly a series of sequential messages and actions. Planning for a supply-chain test bed for this product relies on an understanding of each of these messages and actions, and when they occur. In turn, understanding the messages and actions in the supply chain depends on the sequence of actions within each member of the supply chain. This paper reports on work to model and analyze these messages.

The purpose of the work was to identify the nature of the messages so that the infrastructure and standards required to effect the necessary interactions could be tested. Regarding industrial application of the work, the authors believe that as more electronic assembly is subcontracted a more automated set of electronic messages, use of pervasive infrastructure such as the World-Wide Web, and application of open information-technology standards will prove productive in the commerce of making printed-circuit assemblies.

The ICM project will use these models to identify those Internet-facilitated transactions that will be a part of the ICM test bed. The model also may help to visualize the transactions developed by ICM. Where deemed appropriate, the model may help us to evaluate the ICM business case, and may suggest processes and infrastructure that will improve the flow of information in the supply chain. The models presented herein do not include manufacturing-specific information flows (see conclusions below).

The information flows presented are critical in the identification of stages where data are no longer amenable to change. We note that a change to the identified data elements after passing such a stage often results in scrapping of tooling or partially completed product, and further will usually require a change to the contract or bid and purchase order. Changes to information sets could cause changes to previously quoted printed-circuit-assembly price and delivery from the board-assembly or the bareboard contractors, and subsequent negotiations and product design could delay price and delivery significantly. These stages are the focus of the ICM just-in-time-data delivery.

The model can be used to recommend extensions of the ICM architecture beyond the current industry domain. The model is somewhat dynamic during the life of the ICM project. We expect to revise this model as the ICM test bed evolves and incorporates new interactions. External partner-process improvements will be adopted and modeled where appropriate.

\section{Conclusions}

Identifying and Grouping Product Data: The project conducted scenario workshops during the early phase of the work. These workshops pointed out that frequently there is an unacceptable time delay before manufacturers received sufficient information about the printed-circuit-assembly product to complete the machine tooling and programming. Key data items, such as correlating the component location on the circuit board with their reference designator, often required manual entry from a paper top-assembly drawing. As a result, the data files needed for a prototype-printed-circuit assembly were identified and indexed on a Web page. NISTIR 6320 Internet Commerce for Manufacturing Product Data [1] documented the results of gathering the needed data files. 
Grouping these data within the context of the sequence of their generation and subsequent use resulted in being able to identify their critical just-in-time staging. Since data can be moved with very little time loss, the two-day to two-week delays presently attributed to product information gathering and correlation in the supply chain can be reduced considerably. The ICM Test Bed can be used to develop the methods to move the data so as to realize much of the identified potential time saving.

Building the Test Bed: With knowledge of the data flows, the ICM project can construct the test bed in a top-down manner. Each of the messages and actions identified in the model can be planned, constructed, and executed. Events in the supply-chain sequence will have an entry point in the test bed such that all follow-on development of agents, software, scripts, and business-to-business transactions will be based upon messages that have an identified source and destination. With these needs in mind, the project has paid careful attention to the identification and definition of all supply-chain transactions within the scope of the test bed.

Intra-Enterprise Models: Data staging is manipulating the printed-circuit-assembly-product data to produce partitioned subsets that supply information to specific functions of the manufacturing process. Electronic messaging permits improvements in data staging so that the data preparation for manufacturing can better approximate a just-in-time scenario. The intra-enterprise data are, in the typical manufacturing process, extracted from the design data at one time, and processed for use later during the manufacturing stages. We recognize that such a process may require modification to achieve the desired just-in-time information delivery. The models, Charts 1-4, revealed sets of intra-enterprise data that are stages in the development process of a printed-circuit assembly. We have identified these data staging events in shaded columns on the left side of appropriate models. Examples are the artwork, the solder-mask data, and silk-screen data. Each stage comprises an information milestone.

Compressing the time expended between data preparation and its utilization for building the product allows the design function more time to prepare appropriate data, thereby raising product quality. Data accuracy is a factor in reducing the number of costly changes that design changes necessitate.

Inter-Enterprise Models: The analysis has identified 23 key inter-enterprise transactions among the major functions in the printed-circuit-production process: the original-equipment manufacturer, the printed-circuit assembly assembler, the bare-board producer, the parts supplier, a parts-preparation service, a test fixture supplier, and a stencil supplier. Knowing the nature of the transactions allows testbed designers to provide sufficient process and infrastructure capability to effect the transactions. Table 1 below presents the transactions and Appendix B to this paper presents more detail about them.

Through the use of the Internet, the ICM project will show that inter-enterprise message latency, or data-float time, can be driven to zero-time. That is, when one partner creates and posts any of the 23 messages, it immediately becomes available to the other partner.

\section{Process-Flow-Model Development}

The ICM project has pursued a model-driven approach to identify business roles, the design and manufacturing processes, and the data involved in producing printed-circuit boards and assemblies. The ICM developed the Process-Flow Model according to the process evolution that the ICM Scenario Workshops suggested. These workshops identified three significant processing entities. 
- Original-equipment manufacturer (OEM) — responsible for developing part or all of the design and issuing the purchase order (Chart 1) for the printed-circuit assembly

- Electronics-manufacturing-service provider (EMS) — contracts to build the printed-circuit assembly (Chart 2)

- Board-fabrication shop_-produces photo tools and uses processes unique to printed-circuit assemblies (Chart 3)

The authors added additional entities and an analysis of key entity interactions during development of the process model.

- Parts-supply and parts-preparation functions prepare parts and kit them for insertion into the bare board (Chart 4)

- Test fixture/adapter supplier prepares the fixture and adapter for use in the functional testing of the assembled printed-circuit assembly

- Inter-enterprise transactions among OEM, EMS, bare-board producer, test fixture and adapter supplier, parts supplier, parts-preparation service, and stencil manufacturer (Chart 5)

The modeling process focused first on the events that involve data used within each member of the supply chain. Modeling these events revealed opportunities for data staging. The models were reviewed during meetings of the ICM project, NEMI, and IPC. During model reviews, participants identified variations within the manufacturing flows. These variations were attributed to differences in printedcircuit-assembly products and individual enterprise-planning sequencing. Prior to the second modeling effort, the project analyzed these variations to determine the impact on the flow of information external to the manufacturing operations. Since consistency remained in the external information flows, these became the focus of the second modeling effort. The internal manufacturing-information flows and their variations were then removed from Charts 1,2 , and 3, leaving the critical boundary-manufacturing functions. These resultant models provide insight into printed-circuit-manufacturing activities, serve as a basis for discussions with industry, and help guide ICM-test-bed implementations and demonstrations.

Charts 1 to 5, and supporting descriptive material, are in the Appendix B to this report.

ICM modeling activities include:

- Activity Model (NISTIR 6516) [5]: This model was developed in 1999 based on the IPC/Ecce Activity Model [3]. It is an entity-relationship model (IDEF-0) [24] that describes the activities and relationships that support the manufacture of printed-circuit assemblies.

- Data Staging: The Process-Flow Model, described in this report, uses information from the Activity Model to develop the Data-Staging model presented later in this section of the paper. The model shows 23 key business transactions and data exchanges occurring among trading partners involved in the manufacturing of printed-circuit boards and assemblies. The trading partners include the original-equipment manufacturer (OEM), electronic-manufacturing-service provider (EMS), board fabricator, and various other suppliers.

The model identifies types of message transactions, senders and recipients, and message sequence and content (data) being exchanged among trading partners. The model also describes major activities occurring within an organization or enterprise to produce the sets of information needed 
among trading partners in the ordering and manufacturing of printed-circuit boards and assemblies. By identifying the information sets, and when or where they are needed, the model enables data staging for just-in-time information exchange, which can lead to improved accuracy and product quality, and it can reduce information latency between enterprises to zero. This ICM Flow Model is used to guide implementation and testing of prototype e-business communications among printedcircuit-manufacturing enterprises in the ICM test bed at NIST.

Using the Data-Staging model, ICM is mapping existing and emerging standards to these message transactions to determine where and if these standards could be used to support integration among printed-circuit-manufacturing, trading partners. This includes reviewing standards from the IPC [15], the National Electronics Manufacturing Initiative (NEMI) [16], RosettaNet [17], Open Application Group (OAG) [18], Object Management Group (OMG) [19], CommerceNet (CNet) [20], Electronic Business XML (ebXML) [21], Silicon Graphics Initiative (Si2) [22], and other standards groups.

Each processing flow was refined and documented primarily for use in analyzing the ICM-test bed-toscenario correlation. Each flow is diagramed to focus on the information inputs and the times when various data are utilized. The ICM-test bed expects to develop just-in-time-information distribution, one of the key areas for product-supply time and for delivery of quality products. The interactions identified in Chart 5 are summarized in Table 1 below.

\section{Process-Flow Model}

The process-flow model is presented on Charts 1-4, which are located in the appendix to this report. There is a chart for each key participant in the supply chain.

- Chart 1: To, within, and from the original equipment manufacturer or OEM

- Chart 2: To, within, and from the EMS

- Chart 3: To, within, and from the bare-board supplier

- Chart 4: To, within, and from the discrete-parts supplier, the parts-preparation supplier, and the test-fixture supplier

The process-flow model is a decomposition of events required to complete a function or activity. Most of the benefits of this study should be realized by identifying the last step at which data must be frozen. Essentially, this is identifying and characterizing just-in-time-information delivery for the supply-chain partners.

The processes have not been decomposed further than deemed necessary to identify messages and data needed within the context of the ICM-Test-Bed project. We have not rigorously developed the flows within each partner, such as the assembly-line flows. We have included, primarily, flows of material items, recognizing that these customarily accompany a message item, such as a production-item traveler, or a shipper's tracking-identification bar code. These resultant flow models as presented represent an approach similar to the design-workflow case study presented in [10]. 


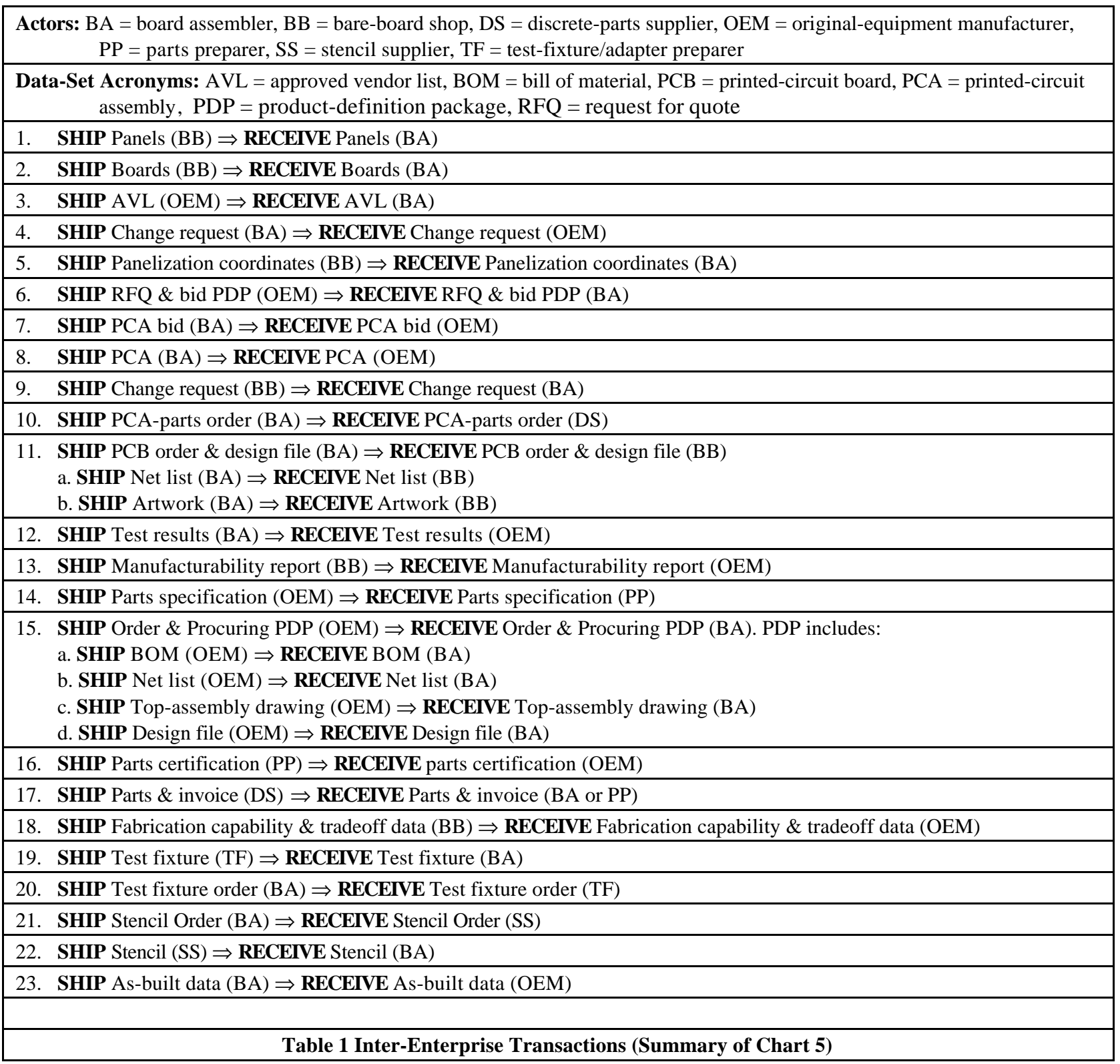

Note: The transaction numbers have no manufacturing significance. They were assigned to maintain tracking consistency during the analysis.

Lines that indicate flow direction connect the sender and receiver of the messages. These connections can be mapped to the ICM-activity model and to the RosettaNet Partner Interface Process (PIP) [17].

The flow model maps readily to the project-activity model that was prepared and documented earlier in the project. The Defense Advanced Research Projects Agency developed that activity model for the Ecce (Electronic Computer-Aided Design to Computer-Aided Manufacturing Exchange) project [3]. In part, the Ecce model was selected because that project has the support of the IPC [15] and the Electronic Industries Association [23]. Based on the Ecce work, the IPC is developing GenCAM (generic computer-aided manufacturing), a CAD-to-CAM, data-transfer standard, also based on the Ecce work. The EIA [23] is developing an extension to their Electronic Data Interchange Format 
(EDIF), to be called CAM-IM, or intermediate format for computer-aided manufacturing of printedcircuit assemblies.

The material in Appendix B describes the functions and information flows within each of the primary partners of the printed-circuit-assembly-supply chain, and finally the messaging between partners. Note that part suppliers, parts-preparation services, test-fixture fabricators, and stencil suppliers participate in the message exchanges. However, the authors have not modeled these internal flows since they are not considered within the scope of the ICM project. Where the notion of control may be useful to the ICM-Test-Bed project, we have identified the function with a note and defined who has control of the information once the information is sent or received.

\section{Transactions between Processes and between Enterprises}

The process-flow models show the principal information-flow routes to procure, produce and test a printed-circuit assembly. From this, the ICM project can achieve parts of two key deliverables, data staging and inter-enterprise transactions.

The transaction occurrence model has to do with improving inter-enterprise transactions. Although all of the information transactions are important, the ones in which the ICM project has the most interest are those that cross enterprise boundaries. These are shown on the Transactions list of Table 1 and in the Transactions-Occurrence Model, or Swim-Lanes model, Chart 5. To design a demonstration, and eventually, an implementation, the project must analyze these transactions for the nature of the information to be exchanged. For example, some of these transactions will be simple electronic-trading transactions such as confirmations, and some will be more sophisticated e-business exchanges such as product-design files. The capabilities should be available where needed to support exchange of the required class of information, including STEP or GenCAM encoded as XML, Gerber files, and native CAD formats. Any proposed solution should not preclude ensuing standards or a wider exploitation of the currently foreseen mechanisms.

\section{Recommendations for Further Research}

The ICM project is similar in scope to some of the in-work initiatives in the National Electronics Manufacturing Initiative (NEMI) organization. The ICM project expects that the model will contribute to various modeling efforts within NEMI activities. As such, the model will likely increase in both quality and visibility. The possible use of the model as a means of examining the emerging NEMI roadmap is open to exploration.

The authors also plan to evaluate the completeness of the scope of the ICM project itself by exploring the possible presentation methods using Web pages and links.

The authors expect that future ICM modeling will develop more detailed process or flow models and data models. The current activities should identify the content and uses of elements of the remaining project models.

Other research should involve analyzing the transactions of Chart 5 and assigning the most appropriate standard, which, if used, will facilitate a successful transaction. 


\section{References and Further Reading}

1. C. Parks, Internet Commerce for Manufacturing Product Data, NISTIR 6320, April 14, 1999.

2. Internet supply chain Partner Interfaces: White Paper, http://www.rosettanet.org/press/white.html RosettaNet Organization URL valid as of April 18, 2000.

3. D. Barton, Electronic CAD to CAM Exchange (Ecce) Project Activity Model, http://www.averstar.com/ecce/ActivityModel/ developed by Averstar (formerly Intermetrics, Inc.). URL valid as of April 18, 2000.

4. IPC-2519, A standard developed by IPC, 2215 Sanders Road, Northbrook, IL 60062-6135.

5. E. Barkmeyer, J. Nell, C. Parks, Internet Commerce for Manufacturing Activity Model, May 3, 2000, NISTIR 6516.

6. T. Costlow, Demo Proves Systems Go for RosettaNet, Electronic Engineering Times, March 13, 2000 , page 87.

7. G. Bartuli, Principles of Configuration Management, Design Engineering, April 1999, page 28.

8. R. Nedbal, Zen and the Art of CAD-to-CAM Data Transfer, Printed Circuit Design, July 1999, page 30.

9. D. Kieran and D. Reed, Practical Approaches to DFM, Printed Circuit Design, December 1998, page 23 .

10. G. Alexander and C Withers, Saving Time; a Workflow Management Success Story, Printed circuit Design, September 1998, page 16.

11. J Gould, CAD \& PDM Integration; Tearing Down the Walls, Design Engineering, September 1998, page 24.

12. Edward Barkmeyer, Neil Christopher, Shaw Feng, James Fowler, Simon Frechette, Albert Jones, Kevin Jurrens, Charles McLean, Mike Pratt, Harry A. Scott, M. K. Senehi, Ram Sriram and Evan Wallace: SIMA Reference Architecture Part I: Activity Models, NISTIR 5939, National Institute of Standards and Technology, Gaithersburg, MD, 1997.

13. F. Bonifazi and J. Gould, Making Workflow Work, Desktop Engineering, April 1998, page 30.

14. J. Henderson, DFM/DFT 101, Basic Rules for Designing Printed Circuit Assemblies, Printed Circuit Design, August 1998, page 12.

15. IPC, formerly Institute for Interconnecting and Packaging Printed Circuits, http://www.ipc.org/, and http://www.gencam.org .

16. National Electronics Manufacturing Initiative (NEMI), http://www.nemi.org

17. RosettaNet, http://www.rosettanet.org

18. Open Application Group ( $\underline{\mathrm{OAG}}$ ), http://www.openapplications.org

19. Object Management Group (OMG), http://www.omg.org

20. CommerceNet ( $\underline{\mathrm{CNet}})$, http://www.commerce.net/

21. Electronic Business XML (ebXML), http://www.ebxml.org/

22. Silicon Graphics Initiative (Si2), http://www.si2.org/ecix/

23. Electronic Industries Association (EIA), http://www.eia.org/

24. IDEF-0, Integrated-Computer-Aided Manufacturing Definition, Activity Model, Integration Definition for Function Modeling, NIST FIPS pub 183, 31 December 1993. Also: Standard Syntax and Semantics for the Integrated Computer Aided Manufacturing (ICAM) Definition Language--Function Modeling (IDEFO), IEEE Standard P1320.1/D6.1 (C/SE), 24 June 1998. 


\section{Appendix A}

\section{The Internet Commerce for Manufacturing Project}

A goal of the ICM project at NIST is to model how the manufacturing industry, through open systems and standards, might participate in this market growth and take advantage of the benefits of Internetbased commerce. In particular, ICM is focusing on the manufacturing of electronic printed circuit boards and assemblies to create an environment where large and small manufacturers of mechanical and electronic components may participate competitively in virtual enterprises that produce complex manufactured systems. ICM collaborates with industry, universities, and standards organizations, to identify and demonstrate solutions for some of the manufacturing information technology problems that exist at the intersection of manufacturing and e-commerce systems.

The beneficiaries of this project are small and medium sized manufacturers of mechanical and electronic components, the large manufacturers of complex systems that incorporate these suppliers into their processes, and software vendors of manufacturing execution systems, enterprise requirements planning systems, and electronic commerce software solutions.

ICM objectives include:

- Enable electronic exchange of build information and change notices for all supply-chain participants to support rapid decision-making and collaboration in the manufacturing and assembly of electronicprinted-circuit boards and assemblies.

- Collaborate with industry and standards organizations to develop public specifications, technologies, and tools for enabling PCB/PCA business and manufacturing operations over the Internet.

- Provide a flexible and neutral test-bed for industry and government to collaborate in testing and evaluating standards-based tools and integration technologies that can reduce the risk of technology adoption by evaluating emerging specifications against various manufacturing scenarios.

- Demonstrate integration of e-commerce business services with technical data interchange for manufacturing processes.

- Disseminate guidance, results and test-bed tools to industry through published papers and the NIST ICM Web site (ICM project: http://www.mel.nist.gov/namt/projects/icm/icm1.htm).

The ICM project is part of the NIST National Advanced Manufacturing Test Bed (NAMT, http://www.mel.nist.gov/namt/) program. ICM is supported by the NIST "Systems Integration for Manufacturing Applications" (SIMA, http://www.mel.nist.gov/msid/sima/sima.htm) program, the Advanced Technology Program (ATP, http://www.atp.nist.gov/atp/), and by participating NIST laboratories (EEEL, MEL, ITL, and MEP). 


\section{Appendix B}

\section{Flow-Diagrams and Transaction Definitions}

On the following pages are Charts 1-5 that diagram the transactions that enter and leave the enterprise during the procurement of printed-circuit assemblies. They are:

- Chart 1: To, within, and from the original equipment manufacturer or OEM

- Chart 2: To, within, and from the EMS

- Chart 3: To, within, and from the bare-board supplier

- Chart 4: To, within, and from the discrete-parts supplier, the parts-preparation supplier, and the test-fixture supplier

- Chart 5: The swim-lane diagram has been created to show only the inter-enterprise transactions among the above enterprises

A legend for Charts 1-5 is presented below.

Decision Transactions

Following are the descriptions of the key transactions among customers and suppliers during printedcircuit-assembly procurement. They are arranged alphabetically and grouped by the various enterprises involved in the procurement.

\section{Assembly Procurement and Electronic-Commerce Flow}

Chart 1: To, within, and from the original equipment manufacturer or OEM

Approve for Update: The OEM reviews the draft engineering-change order for impact upon cost, system performance, and schedule. The designer is notified regarding the changes to be made to the design.

Archive Files: The product-data file is required later in the life cycle of the product, and the file is maintained as an archive. The product-drawing number and version are identified with or are associated with the archived file.

Assemble Buy Order: The procurement request identifies a product identification (including version identification) that is used to obtain the appropriate bid PDP from the product-configuration function. 
Assemble ECO Was/Is and PDP Version: Similar to an order for a new printed-circuit assembly, this consists of an authorization to proceed and a product-definition package (PDP) that contains such items as the bill of material (BOM), the net list, the top-assembly drawing, and the printed-circuitassembly-design file.

Assemble Printed-Circuit-Assembly Order: The order consists of an authorization to proceed and a product-definition package, PDP, that contains such items as the bill of material (BOM), the net list, the top-assembly drawing, and the printed-circuit-assembly-design file. The order also specifies such things as manufacturability report, as-built data, tests and test results, and the design-release file.

AVL: The approved vendor list (AVL) may be included with the Order to specify which vendors are acceptable to the OEM for supplying parts and material for the order.

CAD File/Version*: During the design and layout of a printed-circuit assembly, and for each subsequent change to it, a product-data file is developed on a computer aided design (CAD) system. This file includes the product model defined as two or more conductive layers together with the reference designator for each part, pin numbers, and its associated net name. The product-data file may also include either the generic-part number or the order-part number of each component.

*Note: On completion of a product/version, control of the file is passed to the product-configuration function.

Design Requirements: The process of creating the design requirements is considered outside the scope of the ICM Project. The information from design requirements is the primary input to the printedcircuit assembly designer.

Extract Bill of Materials (BOM)*: The BOM is usually the reference designator of each component together with its assigned part number. The resulting list is often sorted by part number, to which the number of occurrences of each part number is added. Redundant entries of the same part number are then eliminated. A "find" number is assigned to each line in this sorted file.

*Note: On completion of the $\mathrm{BOM}$, control of the BOM is passed to product configuration.

Extract Net List*: The net list consists of the name of each unique signal in the circuit, together with each pin and the reference designator of the pin. For some net list uses, the $\mathrm{X}$ and $\mathrm{Y}$ location of the pin is added into the list.

*Note: On completion of the net list, control of the net list is passed to the product-configuration function.

Output Top-Assembly Drawing*: The top-assembly drawing is primarily a visual used in estimating the production, for tooling, for hand assembly, in troubleshooting, and for support and maintenance documentation. The drawing usually comprises the silk-screen layer, the board outline, and a drawing border.

*Note: On completion of the drawing, control of the drawing is passed to Product Configuration.

Procurement Request: The decision to procure a printed-circuit assembly authorizes a purchasing department to originate a purchase or procurement request. 
Produce ECO: The engineering-change request is evaluated and, if warranted, an engineering-change order $(\mathrm{ECO})$ is originated.

Product Configuration: The product model and supporting documentation are assigned a product identification, or drawing number. Upon review and approval, the identified product-definition package (PDP) is released for acquisition. A make-or-buy review determines if a bid is to be originated. The information needed for an EMS to bid is assembled from selected information (see "Ship Request for Quote") in the PDP. Upon award of an order, the OEM forwards the information needed to manufacture the printed-circuit-assembly version to the EMS. Changes are released as a change order and the "was/is" information is assembled from the PDP and identified by ECO number. When the product-definition package changes the form, fit, or function of the product or part, the productdefinition package is identified as a new version.

See especially reference [1] for examples of acquisition and change-order PDPs that were developed for the ICM Project and posted as Web pages.

Receive As-Built Data: The OEM receives the data from the EMS about what was actually installed on the printed-circuit assembly.

Receive Change Request: During any stage of manufacture suppliers may identify different parts or processes that can either save costs or that are necessary for completion of the manufacturing. Suppliers forward desired changes to the OEM for evaluation by its engineering function.

Receive Fabrication Capability and Tradeoff Data: Should the OEM know the printed-circuitassembly shop at the time of design or re-design, the OEM can use fabrication capability and tradeoff data from the printed-circuit-assembly shop to improve significantly printed-circuit-assembly fabrication yield and production times. This results in lower product costs and higher reliability [8]. This capability information is particularly important for designs operating at higher frequencies or in harsh environments.

Receive Manufacturability Report: The EMS distills information from its control processes, such as statistical-process-control information and supplies it to the OEM. This information is often useful when planning product or design enhancements and for product maintenance. This report also could contain warranty-tracking data, and the as-built BOM.

Receive Part Certification: The parts supplier ships parts that have been qualified to, or modified from, the part specification, together with specification-conformance documentation, to the OEM. The OEM retains the certification as evidence that critical parts meet the specification.

Receive Printed-Circuit Assembly: The OEM receives the final printed-circuit-assembly product from the shipper accompanied by the shipping tracker. The supply-chain process ends upon receipt of the printed-circuit assembly and the invoice, which the OEM forwards to its accounts-payable function.

Receive Printed-Circuit-Assembly Bid: Cost, quantity, and schedule are key data used to evaluate a potential EMS. 
Receive Test Results: The EMS performs tests requested in the request for quote and sends results to the OEM. The test results often facilitate system diagnostic evaluation to identify marginal performance of printed-circuit assemblies.

Select Winning Bid: The OEM reviews quotes received against schedule, cost, and quality requirements and selects a winning bid or several bids. The bid data are used to create a purchase contract.

Ship Order and PDP: The OEM sends the order package to the EMS.

Ship Part Specification: A specification provided by the OEM documents the special process to be applied to procured parts prior to installation on the board. This specification (sometimes called a critical-item specification) details process such as forming, plating, testing, and burn-in.

Ship Request for Quote (RFQ): The OEM reviews procurement request and evaluates capability of potential EMS contractors, and send the RFQ to appropriate EMSs. Alternatively, the OEM posts the RFQ somewhere and notifies the EMSs. For an open bid the OEM publishes the RFQ in an appropriate notification journal. The RFQ includes the "bid PDP" that consists of a top-assembly drawing (or image) and the components to be assembled onto an identified version of the printed-circuit assembly. The printed-circuit-assembly information supplied includes the non-detailed items noted in the Conclusion section of [1], including board outline, overall dimensions, number of layers, solder mask type, hole count, smallest hole size, minimum trace width and spacing, and finish plating.

Note: the printed-circuit-assembly CAD and the photo-plot data are not generally supplied to obtain the printedcircuit-assembly bid.

\section{Board-Assembly Flow}

Chart 2: To, within, and from the EMS.

Printed-Circuit Assembly: Complete all assembly and final preparation operations on the board.

Assemble Part-Buy Order: Accumulate the information required to procure the bare board and the circuit components from the product-configuration function.

Extract Net List: The net list consists of the name of each unique signal in the circuits, together with each pin and the pin-reference designator. For some net-list uses, the $\mathrm{X}$ and $\mathrm{Y}$ location of the pin is added to the list.

Extract PCB: Data needed to process each layer of the board is extracted from the design file, or its neutral representation, as required to create the layer tooling or photo masters.

Extract Printed-Circuit-Assembly Input/Output Signals: The OEM lists signals that are accessible through edge connectors or test points together with the associated pin number and location. This list is required for both the test adapter and the generation of the test program. To prepare for any functional testing when called for in the purchase order, the EMS obtains the list of signals and the connector pin(s) or test point(s) associated with each signal. 
Extract Reference Designators and Locations: To complete either manufacturability analysis, the EMS obtains a list of components, identified by their reference designators, and the component location and orientation.

Extract Test-fixture Data: The information needed to support functional testing is extracted from the $\mathrm{CAD}$ file. These data include the names and connector or test-point locations of signals that participate in the testing. The complete data set, however, usually includes the initialization values of digital strings for each signal, the test vectors for each input signal, and the output vectors or parameters for output signals.

Functional Test: A functional test, when required by the OEM, simulates the actual behavior of the printed-circuit assembly. Power is applied to the printed-circuit assembly, the simulated inputs are applied, and the output responses are measured. Testing may involve signals which are analog, digital, or both.

Kit Parts: The discrete parts from a storeroom must be kitted and formed by the EMS. A kit contains all of the parts to be assembled with one bare board to produce the completed printed-circuit assembly. A parts-preparation service may form and kit the parts in printed-circuit-assembly-sized lots. Exact method of kitting depends upon parameters such as traceability requirements, the assembly equipment present, and the number of assemblies being produced.

Printed-Circuit-Assembly CAD Data: The OEM develops the computer-aided design (CAD) file and transmits a subset to the EMS on award of an order for a printed-circuit assembly. This subset file includes the product model for the printed-circuit-assembly, the reference designators and locations, net list, printed-circuit-assembly input/output signals, and functional-test requirements

Prepare Bid: The EMS prepares a response to the procurement request (RFQ) from the OEM.

Prepare to Ship: A process step in which the PCAs are packaged as required for shipment.

Produce Machine-Insertion Files: These are the robotic-instruction sets for placing parts into the printed-circuit assembly.

Receive Board or Panel*: The EMS receives the bare boards already cut from panels or still in panel form from the bare-board supplier. Also transmitted is some form of documentation that identifies the contents, order and order number or contract number.

*Note: Control of the product shifts to the EMS, however the product may not be modified without permission of the OEM.

Receive Change Request*: The EMS receives a change request from the bare-board supplier. *Note: Control of the request remains with the supplier, however the EMS will forward the request information to the OEM. Depending on the business arrangement, the request could go directly to the OEM or to both the EMS and the OEM, but either way the information transmitted is similar. 
Receive Order/Version: Receive Approved Vendor List (AVL)*: Some or all of the parts are supplier-specified by the OEM. The AVL indicates those manufacturer/vendors approved by the OEM to supply parts listed on the BOM.

*Note: Control of the AVL remains with the OEM, and upon issuing the purchase order the AVL may not be modified without permission of the EMS.

Receive Order/Version: Receive Parts*: The EMS will receive parts from a discrete-parts supplier or a parts-preparation service. The discrete parts will be in lots independent of the needs of the assembly on order and must be kitted and formed by the EMS. The parts-preparation service may form and kit the parts in large printed-circuit-assembly-sized lots.

*Note: Control of the parts shift to the EMS.

Receive Order/Version: Receive Product Data Package (PDP)*: The EMS receives the PDP, which includes the top assembly drawing, the bill of materials (BOM), artwork information for all board layers, and information needed for parts mounting/insertion. This list indicates, for each specified part, the product identification (drawing number), the reference designator for the part, and the supplier identification.

*Note: Control of the PDP remains with the OEM, and upon issuing the purchase order the PDP may not be modified without permission of the EMS.

Receive Panelization Coordinates*: The EMS receives from the bare-board supplier the locations of the assembly layouts on the standard-sized panels so that the EMS can plan the parts-placement function.

*Note: Control of the panel coordinates remains with the board supplier, however the coordinates may not be modified without advising the EMS.

Receive RFQ*: The EMS receives the procurement request from the OEM.

*Note: Control of the request remains with the OEM, however the request may not be modified without permission of the EMS.

Receive Test Fixture: The EMS receives the fixture for the printed-circuit assembly that is to be used to connect the printed-circuit assembly (also known as unit under test) to a testing system during functional testing.

Receive stencil: The EMS receives the stencil for the board notations from the stencil supplier.

Run Functional Test: Where required on the Order, the completed PWA is tested to verify that the functionality requirements are met.

Run Manufacturability Analysis: This test verifies that the conductive patterns that will be produced by the photo tool will correctly implement the net list. The test compares the photo-plotter, inputgeometry data with the net list. This test will identify, for example, nets shorted due to a logotype or part identification rendered in metalization touching more than one active trace.

Ship As-Built Data: The EMS transmits the data about what was actually installed on the printedcircuit assembly to the OEM. 
Ship Change Request: During any stage of manufacture suppliers may identify different parts or processes that can either save costs or that are necessary for completion of the manufacturing. Suppliers forward desired changes to the OEM for evaluation by its engineering function.

Ship Manufacturability Report: The EMS distills information from its control processes, such as statistical-process control information and supplies it to the OEM. This information is often useful when planning product or design enhancements and for product maintenance. This report also could contain warranty-tracking data, and the as-built BOM.

Ship Parts Order: The EMS will order parts from a discrete-parts supplier or a parts-preparation service. The discrete parts will be in lots independent of the needs of the assembly on order and must be kitted and formed by the EMS. A kit contains all of the parts to be assembled with one bare board to produce the completed printed-circuit assembly. There may also be an order to the parts-preparation service to form and kit the parts in printed-circuit-assembly-sized lots.

Ship Printed-Circuit-Board Order/Version: The EMS sends the package that orders the printedcircuit-board to the bare-board supplier. The package contains that part of the product data necessary to fabricate the board layers and test the boards such as the net list and the artwork-defining data.

Ship Printed-Circuit Assembly: The final printed-circuit-assembly product is forwarded to the shipper, and is accompanied by the shipping tracker. An invoice accompanies the product or may, by agreement, be forwarded independent of the product. When the OEM receives the printed-circuit assembly, the invoice is forwarded to the accounts-payable function, and the supply-chain process is ended.

Ship Printed-Circuit-Assembly Bid: The EMS transmits cost, quantity, schedule and other requested information to the OEM.

Ship Stencil Order: The EMS orders the stencil for the board notations from the stencil supplier.

Ship Test-fixture Order: The EMS sends an order to the shop selected to fabricate the test fixtures(s) for the printed-circuit assembly as required for any functional testing specified in the subject printedcircuit assembly order. Included with the test-fixture order are the data specifying the printed-circuitassembly signals subject to test, together with their access locations on the assembly. The characteristics of these signals are also specified, such as input/output/bi-directional, digital/analog, and $\min / \mathrm{max}$ voltage or current.

Ship Test Results: The EMS performs tests requested in the request for quote and sends results to the OEM. The test results often facilitate system diagnostic evaluation to identify marginal performance of printed-circuit assemblies.

Surface-Mount Technology (SMT): Components that mount to the surface of a printed-circuit assembly rather than into holes drilled (called through holes) through the board. 


\section{Board Procurement and ECO Flow}

\section{Chart 3: To, within, and from the bare-board supplier}

Add coupon: A decision is made whether to add a test coupon to the panel. When required either for internal quality control or for customer assurance as stated in the order or contract, the coupon can be removed following panel processing to determine if all processes resulted in desired product quality.

Apply Solder Mask: A process step that covers areas of the board that will not be subsequently solder-coated.

Artwork Data OK: The data needed to produce the photo tool for each layer is checked for errors such as traces not entirely within the bounds of the board, and the existence of correct location of tooling holes (fiducials).

Board-Fabrication Processes: These processes are internal to the fabrication of the bare board. Included are: produce photo-tool data, apply photo-resist, expose and etch, assemble stack-up, press and cure panel, drill panel, clean and plate panel, apply solder mask, solder-coat panel, silk-screen panel, and rout the bare board--full, with tabs, or no.

Drill Panel: A process step in which all holes are drilled (or punched or laser-burned) in the board.

Extract Artwork by Layer: Photo plotter input data is extracted and formatted for the plotting system; one data set for each copper layer to be processed for the complete board/panel.

Extract Drill Data: The location and size is extracted for each hole to be drilled in the processed board/panel.

Extract Printed-Circuit-Assembly-Edge Data: The perimeter coordinates are extracted which will be used to cut, or partially cut, the board from the panel in which it has been fabricated.

Extract Silk-Screen Data: Imaging data is extracted which is to be used to produce a screen which allows ink to be deposited for lettering and other marking of the surface of the board/panel.

Extract Solder-Mask Data: Photo plotter input data is extracted and formatted for plotting the photo tool used to produce a film on the board/panel covering areas where solder is not desired.

Layer X Phototool Data: The data required to produce the specified layer is passed into the boardfabrication process.

Panelize PCBs: Coordinates are defined for the location of one or more printed-circuit boards to be created on a panel which is of a size specified by the fabricator or by his agreement with the contracting EMS.

Receive B-Stage Material: The uncured or partially cured sheet material which is used to insulate and bond two or more details (double sided processed boards) is received for processing into product. 
Receive Printed-Circuit-Assembly Order*: The printed-circuit-assembly-purchase order, together with the product-definition package (PDP), constitute the authorization to schedule production of the product as defined by the PDP.

*Note: Control of the order remains with the OEM, however subsequent modifications to the order, or the PDP may be the basis for a request to re-negotiate the order.

Receive Two-Sided-Board Stock: The copper clad material together with the material/lot specification is received for processing into product.

Rout PWB (or PCA): As specified on the board order, the panel is either sent to be routed (cut from the panel) or the process is skipped and may be accomplished by the EMS at a later stage in completing the PCAs.

Ship Boards: Ship completed boards that have been cut from their standard panels.

Ship Change Request: During any stage of manufacture, different parts or processes may be identified that can either save costs or are necessary for completion of the manufacturing. When identified, and when determined to necessitate a change to the design version, the changes are forwarded to the engineering function for evaluation. In this case the request information flows from the bare-board supplier to the EMS for consideration.

Ship Fabrication Capability/Tradeoff Data: When the printed-circuit-assembly fabricator is known by the OEM at the time of design or re-design, the OEM benefits from learning the assembly fabricator's capability tradeoffs and their cost deltas. The shipping of this information becomes more critical as the printed-circuit-assembly-production run becomes longer and the fabrication rules become tighter. The information may include tolerance capabilities for particular trace shapes that function as passive electronic components (i.e., resistors, capacitors, and inductors).

Ship Panelization Coordinates: The bare-board supplier transmits to the EMS the locations of the board layouts on the standard-sized panels so that the parts-placement function can be planned.

Ship Panels: Ship completed boards that have not been cut from their panels.

Silk Screen Panel: Ink is applied to the panel using a silk screen prepared as a mask, resulting in the labels identifying parts, part number, test points, and other information.

\section{Parts-Supply, Preparation, Stencil, and Test-Fixture Flow}

Chart 4: To, within, and from the discrete-parts supplier, the parts-preparation supplier, and the test-fixture supplier 
Note: Some or all of this function may be performed within the EMS activity. We have flow modeled the completely out-sourced case as a means of identifying messages which could occur formally in the out-sourced situation and would otherwise likely occur in some form. Additional parts-related functions occur within the EMS such as "Place in Inventory" or "Deliver to Production Line" but which may not influence the supply-chain messages.

Burn-in: A part preparation process in which the parts are subjected to specified operating conditions and assures that early-failure parts are eliminated.

Electrical Test: Verify that electrical preparations have been successfully completed.

Extract Electrical Preparation: Extract from the part-specification requirements for making electrical modifications to the parts.

Extract Mechanical Preparation: The part supplier extracts requirements for making mechanical modifications to the parts from the part-specification.

Fit Test: The part supplier mechanically checks part size when required by the part specification.

Laser Trimming: Generally applicable to deposited resistors, material is eroded away until the value matches the part-specification requirement.

Lead Form: Part leads are bent to customer specifications.

Mechanical Inspection: Usually a visual check, the part is given a non-functional inspection.

Package to Customer Specifications: This could be for mounting into a robot-feed system or into assembly-lot kits.

Plating: The parts preparer plates or coats part leads, as needed, for soldering into the printed-circuit assembly.

Presetting: The parts preparer sets the parts that are adjustable, to comply with the parts-specification requirements.

Program ROM: The parts preparer programs read-only-memory devices to tables of values given in the part specification.

Receive Parts: This item applies if the parts-preparation enterprise is a different enterprise from the EMS.

Receive Parts Order: The part supplier receives the order transmitted by the EMS.

Receive Part Specification: Often called a "Critical-Item Specification," this specification both details the preparation of procured parts and, when required, specifies the new part number for part reidentification. 
Receive Stencil Order: The stencil supplier receives the order for the stencil for the board notations from the EMS.

Receive Test-Fixture Order: The shop selected to fabricate the test fixtures(s) for the printed-circuit assembly receives an order from the EMS for test fixtures required for any functional testing specified in the subject printed-circuit assembly. Included with the test-fixture order are the data specifying the printed-circuit assembly signals subject to test, together with their access locations on the board. The characteristics of these signals are also specified, such as input/output/bi-directional, digital/analog, and $\mathrm{min} / \mathrm{max}$ voltage or current.

Re-Identify: The parts specification may require the parts to receive a new identifier after the preparation phase is complete.

Ship Parts and Invoice: This item applies if this enterprise is the parts-supply enterprise to the EMS. Parts may or may not be electrically or mechanically prepared before shipment.

Ship Parts Certification: When required by the part specification, the part supplier prepares a certification is for the part that is shipped to the OEM. Among the items usually required are materialsquality statements, reliability, country of origin, EMF hardness.

Ship Stencil: The stencil supplier ships the stencil to the EMS.

Ship Test Fixture: The test-fixture supplier ships the stencil to the EMS.

Track Identification: The number assigned to a prepared part is associated with the part traveler.

Trimming: The parts preparer cuts the leads to length for mounting. 


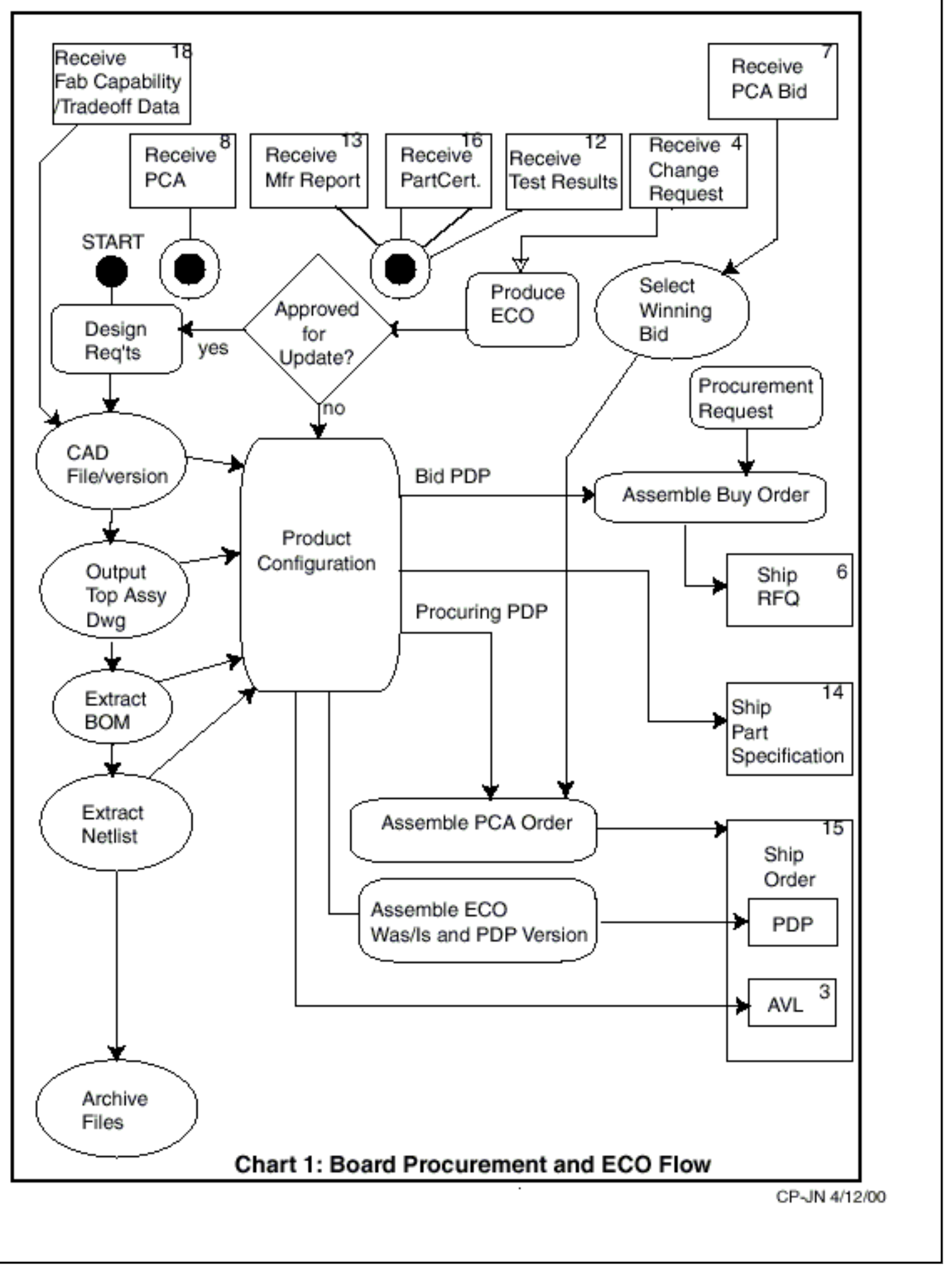




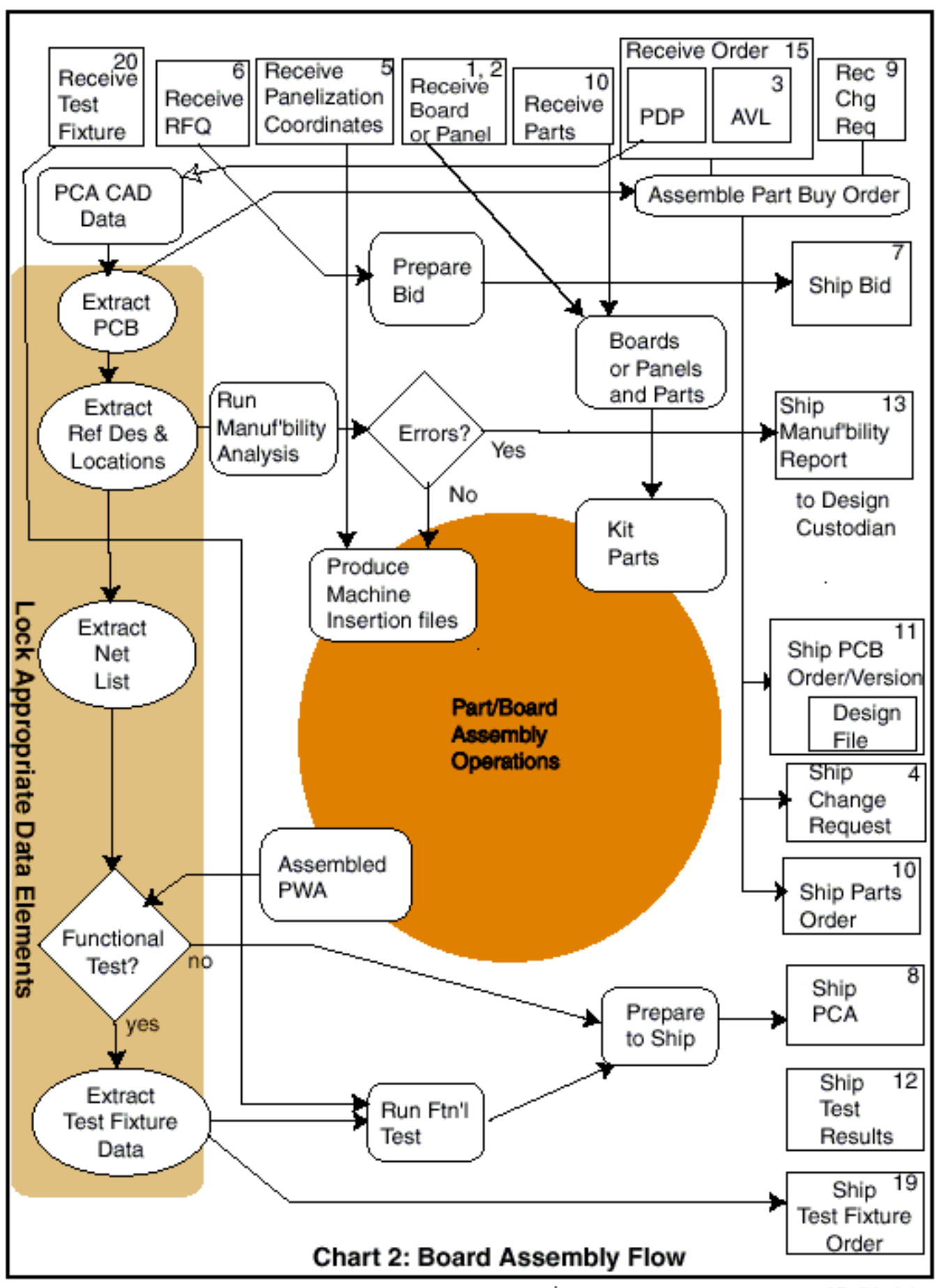

CP-JN 6/1/00 


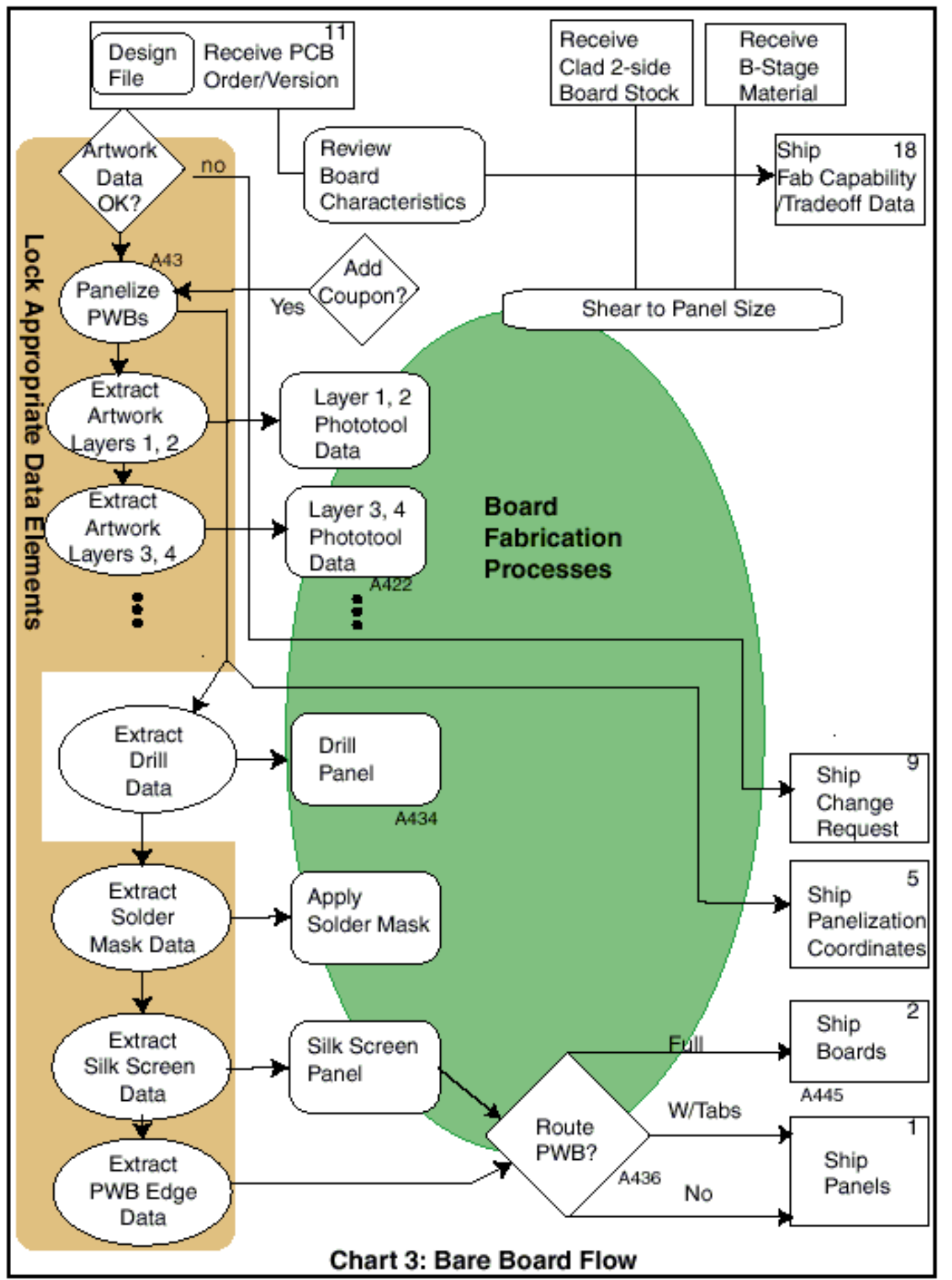

CP $\sqrt{ } N$ 6/1/00 


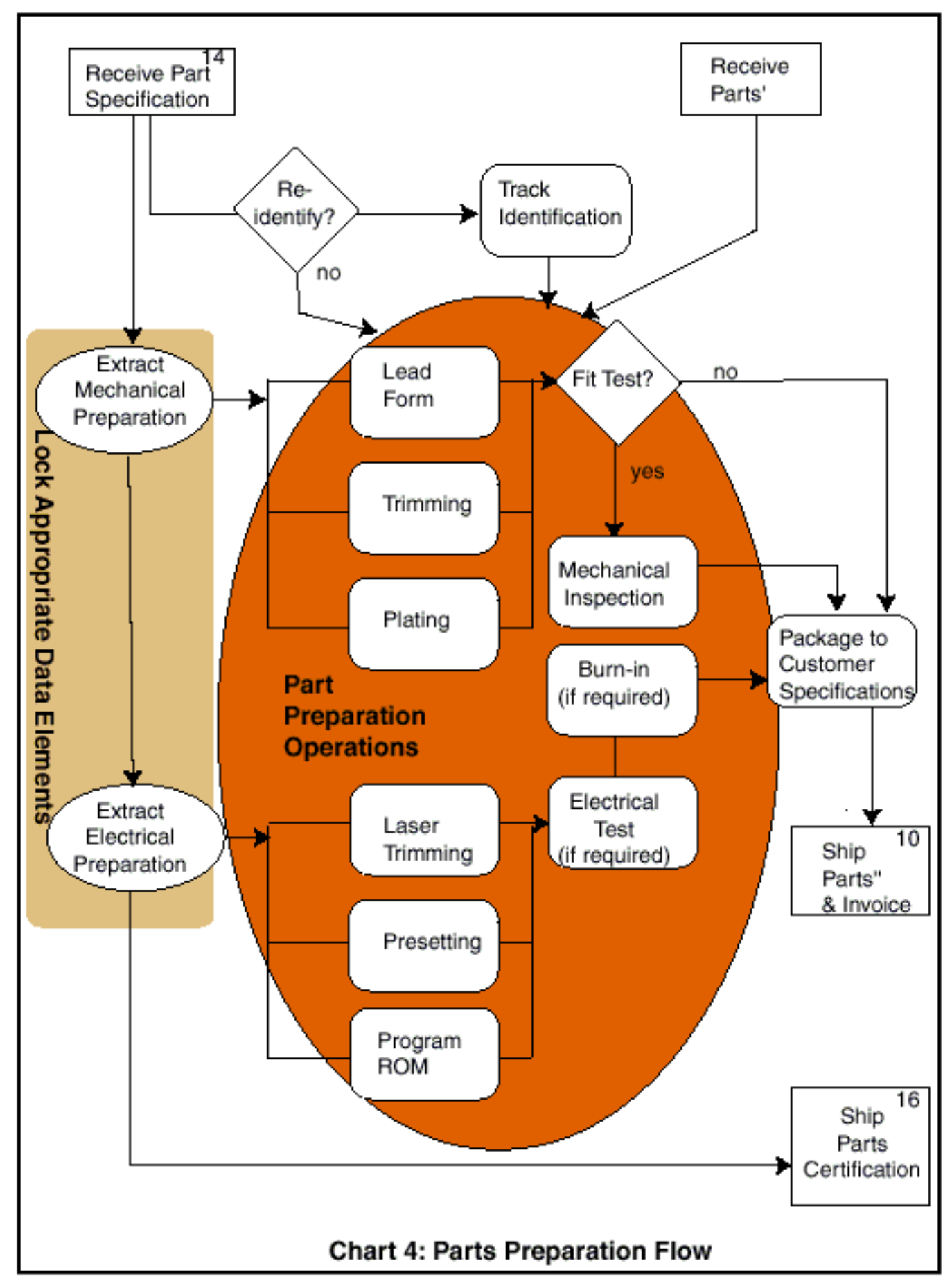

CP $\sqrt{ }$ N 3/30/00 


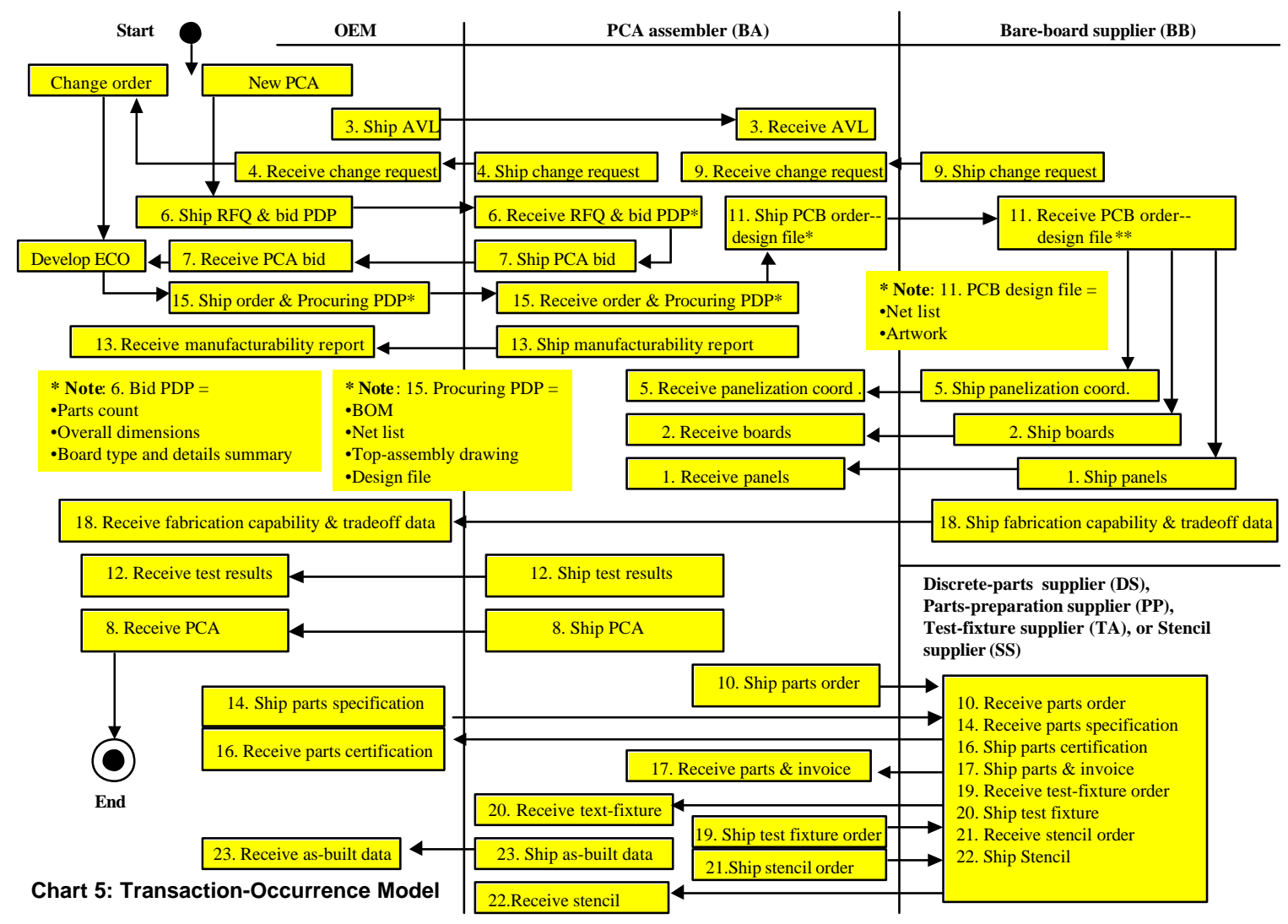

S:jnellicmlswimlanes.ppt 18-Oct-00 